\title{
Synthesis of metallophophaalkenes by reaction of organometallic nucleophiles with a phosphaethynolato-borane
}

Received 00th January 20xx, Accepted 00th January 20xx DOI: $10.1039 / \times 0 \times x 00000 x$

\author{
Daniel W. N. Wilson ${ }^{\mathrm{a}}$ and Jose M. Goicoechea*a
}

We describe the reaction of a phosphaethynolato-borane [B]OCP $\left([\mathrm{B}]=N, N^{\prime}\right.$-bis(2,6-diisopropylphenyl)-2,3-dihydro-1H-1,3,2diazaboryl) with the organometallic nucleophile $\mathrm{Na}\left[\mathrm{Cp} * \mathrm{Fe}(\mathrm{CO})_{2}\right]$ $\left(C p^{*}=\right.$ pentamethylcyclopentadienyl). The electrophilic character of [B]OCP allows for a new route towards the formation metalphosphorus bonds affording a metallophosphaalkene that can be functionalised at both the oxygen and phosphorus atoms depending on the reagents employed.

Phosphaalkynes $(R-C \equiv P)$ are heavier analogues of nitriles ( $R-$ $\mathrm{C} \equiv \mathrm{N}$ ) and, as such, their reactivity is largely dominated by their inherent nucleophilicity. ${ }^{1}$ The parent compound, $\mathrm{H}-\mathrm{C} \equiv \mathrm{P}$; methylidynephosphine (or phosphaacetylene), was first reported in 1950, ${ }^{2}$ and unambiguously characterised in $1961 .^{3}$ Pioneering work on the synthesis and characterization of R$\mathrm{C} \equiv \mathrm{P}$ type compounds by Becker, Nixon, Regitz (and others) culminated with the isolation of the first kinetically stabilized phosphaalkyne ${ }^{t} \mathrm{Bu}-\mathrm{C} \equiv \mathrm{P}$ in $1981 .{ }^{1,4}$ Since then, numerous metal complexes of these interesting species have been isolated, exhibiting a wealth of coordination modes. ${ }^{5}$ In selected cases, the metal centres facilitate oligomerization of the phosphaalkynes to give rise to cyclic species such as diphosphacyclobutadienes and 1,3,5-tri-phosphabenzene derivatives. $^{6}$ More recently, the nucleophilicity of phosphaalkynes was exploited to afford $1 \mathrm{H}$-phosphindoles by protonation of a ruthenium complex containing $\left(\mathrm{C}_{6} \mathrm{H}_{5}\right)_{3} \mathrm{C}-\mathrm{C} \equiv \mathrm{P}$. $^{7}$

Much less explored is the reactivity of phosphaalkynes as electrophiles. The reverse polarity of the carbon-pnictogen bond relative to nitriles, i.e. ${ }^{\delta-} \mathrm{C} \equiv \mathrm{P}^{\delta+}$ vs. ${ }^{\delta+} \mathrm{C} \equiv \mathrm{N}^{\delta-}$, suggests that nucleophilic reagents should react with the phosphorus atom. Cowley and co-workers explored this hypothesis by reacting Mes*-C $\equiv$ P (Mes* $=2,4,6-{ }^{-} \mathrm{Bu}_{3} \mathrm{C}_{6} \mathrm{H}_{2}$ ) with MeLi and were able to show that the methyl functionality associates with the phosphorus centre to afford $\mathrm{ArHC}=\mathrm{PMe}$ (after addition of $\mathrm{H}_{2} \mathrm{O}$ to the reaction mixture). ${ }^{8}$ However, to our knowledge, this

\footnotetext{
a. Department of Chemistry, University of Oxford, Chemistry Research Laboratory, 12 Mansfield Road, Oxford, OX1 3TA, U.K.

E-mail: jose.goicoechea@chem.ox.ac.uk

Electronic Supplementary Information (ESI) available: Experimental and analytical data for all compounds. See DOI: 10.1039/x0xx00000x
}

report constitutes one of the few examples where the reactivity of phosphaalkynes towards nucleophiles has been explored as a way of accessing novel organophosphorus compounds. ${ }^{9}$

We recently reported on the isolation of a novel phosphaethynolato-borane by reaction of a cyclic bromoborane with sodium salts of the phosphaethynolate anion $\left(\mathrm{PCO}^{-}\right) \cdot{ }^{10,11}$ This species, $[\mathrm{B}] \mathrm{OCP}\left([\mathrm{B}]=N, N^{\prime}\right.$-bis $(2,6-$ diisopropylphenyl)-2,3-dihydro- $1 H$-1,3,2-diazaboryl), is valence isoelectronic with phosphaalkynes and formally contains a $\mathrm{C} \equiv \mathrm{P}$ triple bond. [B]OCP is unstable in the presence of Lewis bases leading to dimerization in donor solvents (such as THF). Moreover, we demonstrated that $[B] O C P$ reacts with strong nucleophiles such as LiMes with concomitant functionalization at the phosphorus atom. These findings suggest that $[B] O C P$ is significantly electrophilic. This is due to a low-lying LUMO with $\pi^{*}$ character and a significant contribution from a phosphorus atom p-orbital. Herein we report some preliminary findings exploiting this reactivity pathway, which have led to a novel way of accessing metallophosphaalkenes. ${ }^{12}$

While [B]OCP can be considered a phosphaalkyne, its physical and chemical properties differ from that of more classical $\mathrm{R}-\mathrm{C} \equiv \mathrm{P}$ systems (where $\mathrm{R}=\mathrm{H}$, alkyl or aryl). For example, the ${ }^{31} \mathrm{P}\left\{{ }^{1} \mathrm{H}\right\}$ NMR resonance observed for [B]OCP ($285.9 \mathrm{ppm})$ is notably upfield compared to ${ }^{\mathrm{t}} \mathrm{Bu}-\mathrm{C} \equiv \mathrm{P}(-69.2$ ppm) and $\mathrm{H}-\mathrm{C} \equiv \mathrm{P}(-32 \mathrm{ppm}), 4,13$ and even more so when compared to silyl- or aryl-functionalised species such as $\mathrm{Me}_{3} \mathrm{Si}-$ $\mathrm{C} \equiv \mathrm{P}(+96 \mathrm{ppm}) \cdot{ }^{14}$ This chemical shift, and the structure of $[\mathrm{B}] \mathrm{OCP}$, in which the boryl ring and the phosphaethynolate moiety are co-planar, indicate a significant degree of electron delocalization over the totality of the [B]OCP $\pi$-manifold. This results in greater electropositive character at the phosphorus atom, implying that $[\mathrm{B}] \mathrm{OCP}$ is a relatively strong electrophile.

We set out to probe this hypothesis by reacting [B]OCP with the archetypal transition metal reagent $\mathrm{Na}\left[\mathrm{Cp} * \mathrm{Fe}(\mathrm{CO})_{2}\right]$ $\left(\mathrm{Cp}^{*}=\right.$ pentamethylcyclopentadienyl), which is known to act as a nucleophile in, for example, $\mathrm{S}_{\mathrm{N}}{ }^{2}$ reactions. ${ }^{15}$ The reaction of [B]OCP with one equivalent of $\mathrm{Na}\left[\mathrm{Cp} * \mathrm{Fe}(\mathrm{CO})_{2}\right]$ was found to afford a novel anionic metallophosphaalkene in which the $\mathrm{Cp} * \mathrm{Fe}(\mathrm{CO})_{2}$ unit $\left(\mathrm{Fp}^{*}\right)$ associates with the phosphorus centre 
to afford $\{[\mathrm{B}](\mathrm{NaO}) \mathrm{C}=\mathrm{PFp} *\}_{2}\left(\{\mathrm{Na}[\mathbf{1}]\}_{2}\right.$; as pictured in Scheme $1)$. To the best of our knowledge, this reaction is the first example of a phosphaalkyne being used as an electrophile for the formation of a metal-phosphorus bond. ${ }^{16}$

Conversion to 1 was observed in the ${ }^{31} \mathrm{P}\left\{{ }^{1} \mathrm{H}\right\}$ NMR spectrum of the reaction mixture which exhibits a downfield-shifted singlet resonance at $188.6 \mathrm{ppm}$. The ${ }^{1} \mathrm{H}$ NMR spectrum in consistent with the presence of one $\mathrm{Fp}^{*}$ and one boryl moiety, while the ${ }^{13} \mathrm{C}\left\{{ }^{1} \mathrm{H}\right\}$ NMR spectrum reveals a single resonance at $222.05 \mathrm{ppm}$ for the central carbonyl carbon atom, shifted relative to that of the $[B] O C P$ precursor $(140.15 \mathrm{ppm})$. It is noteworthy that in this reaction, as in the reaction with LiMes, the boryl group migrates from the oxygen to the carbon atom of the phosphaethynolate.

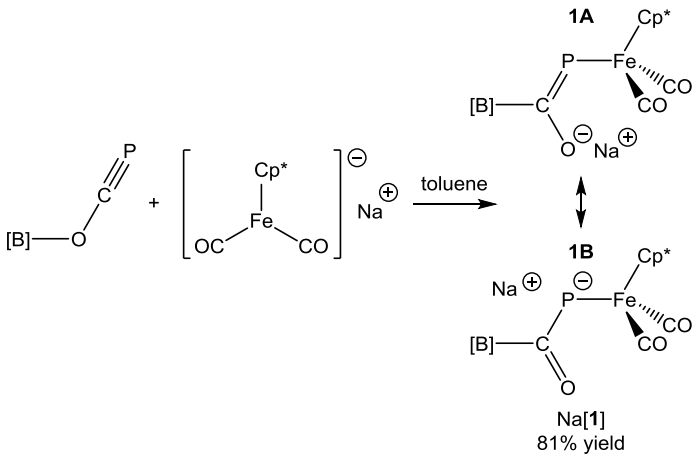

Scheme 1. Synthesis of $\mathrm{Na}[1]$ from reaction of $[\mathrm{B}] \mathrm{OCP}$ with $\mathrm{Na}\left[\mathrm{Cp} * \mathrm{Fe}(\mathrm{CO})_{2}\right]$. [B] $=N, N^{\prime}-$ bis(2,6-diisopropylphenyl)-2,3-dihydro-1H-1,3,2-diazaboryl.

Slow evaporation of a toluene solution of the reaction mixture afforded red crystals of $\{\mathrm{Na}[1]\}_{2}$ suitable for single crystal X-ray diffraction in high yield (81\%). The single crystal structure of $\{\mathrm{Na}[1]\}_{2}$ reveals a tight ion pair featuring two metallophosphaalkene moieties bridged by the same number of sodium cations. For clarity bond metric data will only be discussed for one of these units (Figure 1).

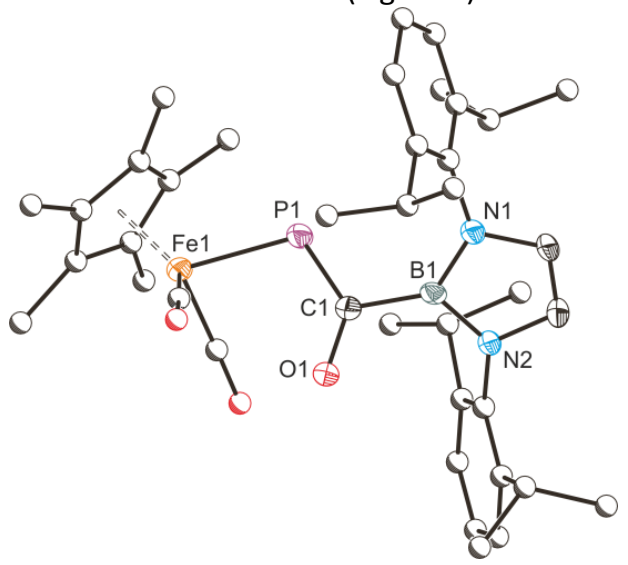

Figure 1. Molecular structure of 1. Anisotropic displacement ellipsoids set at 50\% probability. Hydrogen atoms have been omitted for clarity. Atoms of the Fp* moiety (with the exception of $\mathrm{Fe}$ ) and the Dipp groups are pictured as spheres of arbitrary radius. Selected interatomic distances $[\AA \AA]$ and angles $\left[^{\circ}\right]$ : Fe1-P1 2.322(1), P1-C1 1.725(3), C1-O1 1.315(3), C1-B1 1.578(4), B1-N1 1.438(4), B1-N2 1.436(4); Fe1-P1-C1 111.03(9), P1-C1-O1 129.7(2), P1-C1-B1 113.68(19), O1-C1-B1 116.4(2), C1-B1-N1 130.8(3), C1-B1-N2 124.9(3), N1-B1-N2 104.2(2).

The $\mathrm{C}=\mathrm{P}$ bond in $\mathbf{1}$ is significantly elongated $(1.725(3) \AA)$ relative to that of $[B] O C P(1.545(2) \AA)$. This distance falls between the values expected for a $\mathrm{P}-\mathrm{C}$ single $(1.86 \AA$ ) and double bond (1.69 $\AA$ ). ${ }^{17,18}$ The sum of bond angles at the central carbon atom $\left(359.8^{\circ}\right)$ is consistent with a planar, formally $\mathrm{sp}^{2}$ hybridised, atom. $\mathbf{1}$ can be formally discussed as an anionic metallophosphaalkene with a formal negative charge on the oxygen atom (resonance form 1A in Scheme 1), or conversely as a phosphide bearing an acyl substituent (resonance form 1B). Subsequent reactivity studies reveal that it can react as either depending on the reagent in question.
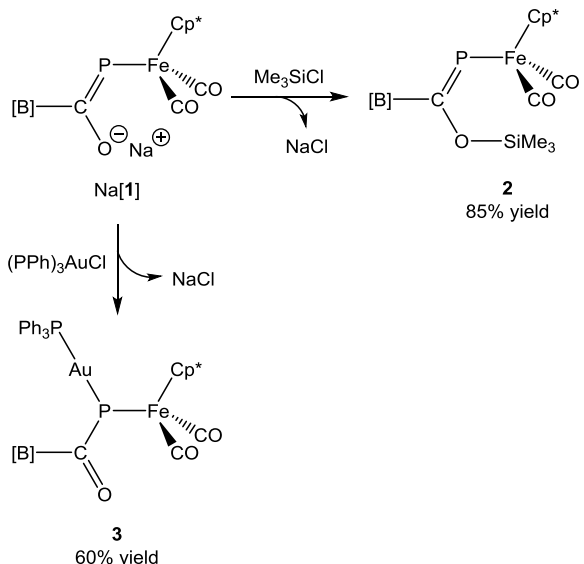

Scheme 2. Metathesis reactions of $\mathrm{Na}[\mathbf{1}]$ on reaction with $\mathrm{Me}_{3} \mathrm{SiCl}$ to afford $\mathbf{2}$ and with $\left(\mathrm{PPh}_{3}\right) \mathrm{AuCl}$ to afford 3 .

For example, compound $\mathbf{1}$ is readily silylated by reaction with $\mathrm{Me}_{3} \mathrm{SiCl}$ affording a neutral metallophosphaalkene, $[\mathrm{B}]\left(\mathrm{Me}_{3} \mathrm{SiO}\right) \mathrm{C}=\mathrm{PFp}^{*}(\mathbf{2})$, in quantitative yield (according to Scheme 2). The ${ }^{31} \mathrm{P}$ NMR spectrum reveals a singlet resonance at $350.6 \mathrm{ppm}$ (cf. $188.6 \mathrm{ppm}$ for $\mathrm{Na}$ [1]) which is consistent with other literature-reported phosphaalkenes. For example, the closely related species ${ }^{t} \mathrm{Bu}\left(\mathrm{Me}_{3} \mathrm{SiO}\right) \mathrm{C}=\mathrm{PFp}\left(\mathrm{Fp}=\mathrm{CpFe}(\mathrm{CO})_{2}\right)$ exhibits a singlet resonance in its ${ }^{31} \mathrm{P}\left\{{ }^{1} \mathrm{H}\right\}$ NMR spectrum at $215.2 \mathrm{ppm},{ }^{19}$ while $\left(\mathrm{Me}_{3} \mathrm{Si}\right)_{2} \mathrm{C}=\mathrm{PFp}$ is observed at $641.5 \mathrm{ppm} .{ }^{20}$ The ${ }^{1} \mathrm{H}$ NMR spectrum largely remains unchanged with the exception of an additional resonance at $0.15 \mathrm{ppm}$ from the trimethylsilyl group. Red crystals of $\mathbf{2}$ could be isolated from a concentrated hexane solution in $85 \%$ yield (Figure 2 ).

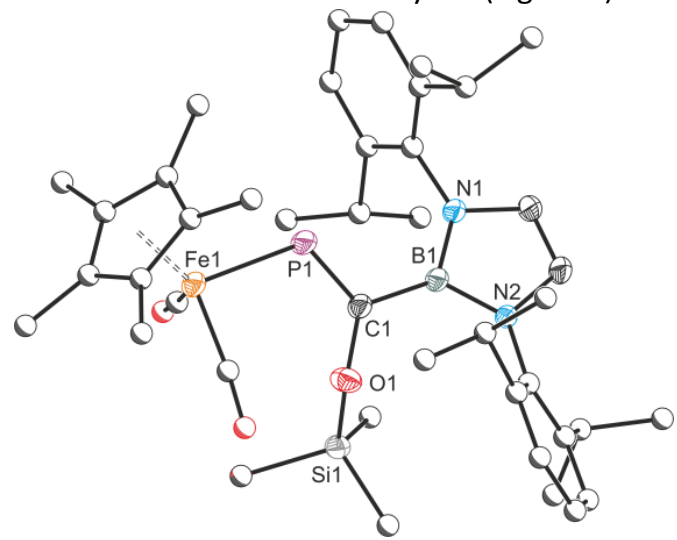

Figure 2. Molecular structure of 2. Anisotropic displacement ellipsoids set at $50 \%$ probability. Hydrogen atoms have been omitted for clarity. Atoms of the Fp* moiety (with the exception of $\mathrm{Fe}$ ) and the Dipp groups are pictured as spheres of arbitrary radius. Selected interatomic distances $[\AA ̊]$ and angles [ $\left[^{\circ}\right]$ : Fe1-P1 2.2738(7), P1-C1 1.694(2), C1-O1 1.381(3), O1-Si1 1.651(2), C1-B1 1.568(3), B1-N1 1.440(3), B1-N2 1.448(3); Fe1-P1-C1 115.29(9), P1-C1-O1 124.10(17), P1-C1-B1 116.80(18), O1-C1-B1 118.5(2), C1-B1-N1 127.0(2), C1-B1-N2 128.8(2), N1-B1-N2 104.2(2). 
Structurally there is no significant change to the metallophosphalkene core of $\mathbf{1}$ on silylation. There is a slight shortening of the C-P bond in 2 (1.694(2) compared to $1.725(3) \AA$ in $\left.\{\mathrm{Na}[1]\}_{2}\right)$, which is a result of increased localisation of electron density in the bond C-P $\pi$ bond. This is accompanied by a shortening of the Fe-P bond on loss of any delocalised negative charge on the phosphorus atom.

Prompted by the results obtained on silylation of $\mathbf{1}$, we explored the reactivity of this species towards other electrophiles that would preferentially bind to the phosphorus atom. Reaction of $\mathrm{Na}[\mathbf{1}]$ with one equivalent of $\left(\mathrm{PPh}_{3}\right) \mathrm{AuCl}$ gives rise to a novel species (3) exhibiting two doublet resonances of equal intensity in the ${ }^{31} \mathrm{P}\left\{{ }^{1} \mathrm{H}\right\} \mathrm{NMR}$ spectrum at 98.8 and $43.3 \mathrm{ppm}\left({ }^{2} \mathrm{~J}_{\mathrm{p}-\mathrm{p}}=102.7 \mathrm{~Hz}\right)$. The former of these corresponds to the phosphide substituent $\mathrm{P}\{\mathrm{C}(\mathrm{O})[\mathrm{B}]\} \mathrm{Fp}^{*-}$, while the latter is due to the $\mathrm{PPh}_{3}$ ligand coordinated to the gold centre. Recrystallisation of this compound from a concentrated toluene solution afforded orange crystals of a linear gold(I) compound in moderate yield (60\%).

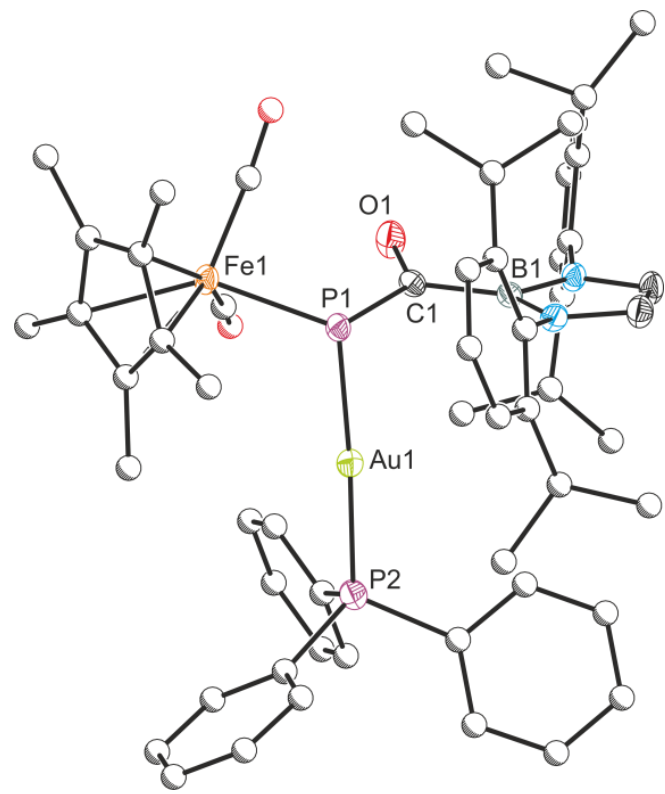

Figure 3. Molecular structure of 3. Anisotropic displacement ellipsoids set at $50 \%$ probability. Hydrogen atoms have been omitted for clarity. Atoms of the Fp* moiety (with the exception of $\mathrm{Fe}$ ), $\mathrm{Ph}$ and Dipp groups are pictured as spheres of arbitrary radius. Selected interatomic distances $[\AA \AA]$ and angles [ ${ }^{\circ}$ ]: Au1-P1 2.3282(10), Au1-P2 2.2993(11), Fe1-P1 2.3062(12), P1-C1 1.846(4), C1-O1 1.235(5), C1-B1 1.590(6), B1N1 1.441(5), B1-N2 1.430(5); P1-Au1-P2 175.51(4), Fe1-P1-C1 107.89(14), P1-C1-O1 123.3(3), P1-C1-B1 118.1(3), O1-C1-B1 118.4(3), C1-B1-N1 130.9(3), C1-B1-N2 124.2(4), N1-B1-N2 104.7(3), Au1-P1-Fe1 107.52(4), Au1-P1-C1 94.02(13), Fe1-P1 C1 107.89(14).

The single crystal X-ray structure of $\mathbf{3}$ (Figure 3 ) confirms the presence of a linear gold(I) complex bonded to an anionic phosphido ligand and triphenylphosphine (P1-Au1-P2 $\left.175.51(4)^{\circ}\right)$. In contrast to 2 , which can be rationalized as a bone fide metallophosphaalkene, in $\mathbf{3}$ there is a significant lengthening of the C-P bond (1.846(4) $\AA$; cf. 1.694(2) $\AA$ in 2) in line with the value expected for a single bond. ${ }^{17,18}$ The goldphosphorus bond distances are quite similar (Au1-P1 2.328(1) and Au1-P2 2.299(1) Å) with the bond to the triphenylphosphine substituent being somewhat shorter, presumably due to the presence of a stereochemically active lone-pair on the $\mathrm{P}\{\mathrm{C}(\mathrm{O})[\mathrm{B}]\} \mathrm{Fp}^{*-}$ substituent. The phosphide $\mathrm{P} 1$ atom is pyramidal with a sum of bond angles of $309^{\circ}$. Related gold(I) phosphane/phosphanido complexes, ${ }^{21,22}$ such as $\left(\mathrm{PPh}_{3}\right) \mathrm{AuP}\left(\mathrm{SnMe}_{3}\right)_{2}$, have been found to exhibit comparable bond metrics with the bond to the phosphide functionality (2.320(1) A) being slightly longer than that to the phosphine (2.293(1) Å). ${ }^{22}$

To conclude, we describe a novel route for the formation of metallophosphaalkenes by exploiting the electrophilic character of a unique phosphaalkyne. Migration of the boryl functionality from the oxygen to the carbon atom in [B]OCP allows for the stabilization of anionic phosphaalkenes which can be further functionalized by reactions with electrophiles.

\section{Conflicts of interest}

There are no conflicts to declare.

\section{Acknowledgements}

We thank the EPSRC and the University of Oxford for financial support of this research (DTA studentship D.W.N.W.) and the University of Oxford for access to Chemical Crystallography and Advanced Research Computing (ARC) facilities (http://dx.doi.org/10.5281/zenodo.22558). We also acknowledge Simon Aldridge and Robert Mangan for the donation of $\mathrm{Na}\left[\mathrm{Cp} * \mathrm{Fe}(\mathrm{CO})_{2}\right]$.

\section{References}

1 R. Appel, F. Knoll, and I. Ruppert, Angew. Chem. Int. Ed. Engl. 1981, 20, 731-744.

2 H. Albers, Angew. Chem. 1950, 62, 451.

3 T. E. Gier, J. Am. Chem. Soc. 1961, 83, 1769.

4 (a) G. Becker, G. Gresser and W. Uhl, Z. Naturforsch., B 1981, 38, 16-19; (b) W. Rosch, U. Hees and M. Regitz, Chem. Ber. 1987, 120, 1645 .

5 (a) J. F. Nixon, Chem. Rev. 1988, 88, 1327-1362; (b) J.f Nixon, Chem. Soc. Rev. 1995, 24, 319-328; (c) J. F. Nixon, Coord. Chem. Rev. 1995, 145, 201-258.

6 (a) M. Regitz and P. Binger Angew. Chem. Int. Ed. Engl. 1988, 27, 1484-1508; (b) M. Regitz, Chem. Rev. 1990, 90, 191-213.

7 J. G. Cordaro, D. Stein and H. Grützmacher, J. Am. Chem. Soc. 2006, 128, 14962-14971.

8 A. M. Arif, A. R. Barron, A. H. Cowley and S. W. Hall, J. Chem. Soc., Chem. Commun. 1988, 171-172.

9 (a) D. E. Hibbs, C. Jones and A. F. Richards, J. Chem. Soc. Dalton Trans. 1999, 3531-3532; (b) C. Jones and A. F. Richards, J. Organomet. Chem. 2002, 645, 256-261; (c) S. Ito, H. Sugiyama and M. Yoshifuji, Chem. Commun. 2002, 17441745.

10 D. W. N. Wilson, A. Hinz, and J. M. Goicoechea, Angew. Chem. Int. Ed. 2018, 57, 2188-2193.

11 For recent reviews on the chemistry of the 2phosphaethynolate anions see: (a) J. M. Goicoechea and H. Grützmacher, Angew. Chem. Int. Ed. 2018, 57, 16968-16994; (b) L. Weber, Eur. J. Inorg. Chem. 2018, 2018, 2175-2227.

12 For reviews on metallophosphaalkenes see: (a) L. Weber, Coord. Chem. Rev. 2005, 249, 741-763; b) L. Weber, Angew. Chem. Int. Ed. Engl. 1996, 35, 271-288. 
13 S. P. Anderson, H. Goldwhite, D. Ko, A. Letsou and F. Esparza, J. Chem. Soc., Chem. Commun. 1975, 744-745.

14 R. Appel, and A. Westerhaus, Tetrahedron Lett. 1981, 22, 2159-2160.

15 R. B. King, Acc. Chem. Res. 1970, 3, 417-427.

16 A. Chirila, R. Wolf, J. C. Slootwega and K. Lammertsma, Coord. Chem. Rev. 2014, 270-271, 57-74

17 (a) P. Pyykkö, M. Atsumi, Chem. Eur. J. 2009, 15, 186-197; (b) P. Pyykkö, M. Atsumi, Chem. Eur. J. 2009, 15, 12770-12779.

18 B. Cordero, V. Gómez, A. E. Platero-Prats, M. Revés, J. Echeverría, E. Cremades, F. Barragán, and S. Alvarez, Dalton Trans. 2008, 2832-2838.

19 L. Weber, K. Reizig, R. Boese and M. Polk, Angew. Chem. Int. Ed. Engl. 1985, 24, 604-605.

20 D. Gudat, E. Niecke, A. M. Arif, A. H. Cowley and S. Quashie, Organometallics 1986, 5, 593-595.

21 (a) R. B. Bedford, A. H. Hill, C. Jones, A. J. P. White, D. J. Williams and J. D. E. T. Wilton-Ely, Chem. Commun. 1997 179-180; (b) D. M. Stefanescu, H. F. Yuen, D. S. Glueck, J. A. Golen and A. L. Rheingold, Angew. Chem. Int. Ed. 2003, 42, 1046-1048; (c) S. Gómez-Ruiz, R. Wolf, S. Bauer, H. Bittig, A. Schisler, P. Lönnecke and E. Hey-Hawkins, Chem. Eur. J. 2008, 14, 4511-4520.

22 C. C. Cummins, C. Huang, T. J. Miller, M. K. Reintinger, J. M. Stauber, I. Tannou, D. Tofan, A. Toubaei, A. Velian and G. Wu, Inorg. Chem. 2014, 53, 3678-3687. 